\title{
Enhanced molecular-based surveillance and source attribution of campylobacter infections in the UK
}

Area of research interest: Antimicrobial resistance

Study duration: 2015-10-01

Project status: Completed

Project code: FS101013

Conducted by: Oxford University

Date published: 27 July 2021

DOI: https://doi.org/10.46756/sci.fsa.ksj135

\section{Background}

Campylobacter is the commonest cause of bacterial gastroenteritis among human populations in the developed world, with an estimated 250,000 cases in England and Wales annually. As a consequence, campylobacteriosis is recognised as a major public health concern. There are 40 species and subspecies of Campylobacter recognised to date, of which, $\mathrm{C}$. jejuni accounts for $90 \%$ of identified campylobacteriosis in humans, with C. coli and occasionally C. lari and C. upsaliensis causing the majority of the remaining cases.

\section{Research approach}

This human campylobacteriosis sentinel surveillance project was based at two sites in Oxfordshire and North East England, chosen to be representative of the English population on the Office for National Statistics urban-rural classification and to provide continuity with genetic surveillance started in Oxfordshire in October 2003. Between October 2015 and September 2018 epidemiological questionnaires and genome sequencing of isolates from human cases was accompanied by sampling and genome sequencing of isolates from possible food animal sources.

The principal aim was to estimate the contributions of the main sources of human infection and to identify any changes over time. An extension to the project focussed on antimicrobial resistance in study isolates and older archived isolates. The aim of this additional work was to analyse the emergence of the antimicrobial resistance that is now present among human isolates and to describe and compare antimicrobial resistance in recent food animal isolates.

\section{Results}

Using these approaches, the study estimated that approximately $70 \%$ of Campylobacter jejuni and just under $50 \%$ of $\mathrm{C}$. coli infection was linked to chicken as the source and that this was relatively stable over time. Ruminants were identified as the second most common source for $\mathrm{C}$. jejuni and the most common for $\mathrm{C}$. coli, where there was also some evidence for pig as a source although less common than ruminant or chicken.

With regards to antimicrobial resistance, the study revealed a rise in fluoroquinolone and tetracycline resistance in C. jejuni isolates from human infections between 1997 and 2018. 
Fluoroquinolone resistance was more frequent in C. jejuni isolates from chicken than from other animals, whilst tetracycline resistance was more frequent in poultry and pig isolates than ruminants. Resistance to macrolides and aminoglycosides remain low.

As part of the project, a data storyboard was created and can be viewed online.

\section{Research report}

PDF

View Enhanced molecular-based surveillance and source attribution of campylobacter infections in the UK as PDF(Open in a new window) (4.13 MB) 